\title{
The impact of selected risk factors on the occurrence of highly pathogenic avian influenza in commercial poultry flocks in Poland
}

\author{
Anna Gierak ${ }^{1 凶}$, Krzysztof Śmietanka $^{2}$ \\ ${ }^{1}$ Department of Epidemiology and Risk Assessment, \\ ${ }^{2}$ Department of Poultry Diseases, National Veterinary Research Institute, 24-100 Puławy, Poland \\ anna.gierak@piwet.pulawy.pl
}

Received: August 5, $2020 \quad$ Accepted: February 3, 2021

\begin{abstract}
Introduction: Introduction of highly pathogenic avian influenza virus (HPAIV) into a country and its further spread may have a devastating impact on the poultry industry and lead to serious economic consequences. Various risk factors may increase the probability of HPAI outbreak occurrence but their relative influence is often difficult to determine. The study evaluates how the densities of selected poultry species and proximity to the areas inhabited by wild birds impacted HPAI outbreak occurrence during the recently reported epidemics in Poland. Material and methods: The analysis was developed using these risk factors in the locations of affected and randomly chosen unaffected commercial farms. Generalised linear and non-linear models, specifically logistic regression, classification tree and random forest, were used to indicate the most relevant risk factors, to quantify their association with HPAI outbreak occurrence, and to develop a map depicting spatial risk distribution. Results: The most important risk factors comprised the densities of turkeys, geese and ducks. The abundance of these species of poultry in an area increased the probability of HPAI occurrence, and their farming intensity in several areas of central, western, eastern and northern Poland put these areas at the highest risk. Conclusion: The results may improve the targeting of active surveillance, strengthen biosecurity in the areas at risk and contribute to early detection of HPAI in outbreak reoccurrences.
\end{abstract}

Keywords: poultry diseases, risk map, spatial analysis.

\section{Introduction}

Highly pathogenic avian influenza (HPAI) is an infectious viral disease affecting wild and domestic bird species worldwide (4). The HPAI viruses (HPAIV) emerge from low-pathogenic precursor viruses (lowpathogenic avian influenza viruses, LPAIV) upon transmission from wild aquatic birds and circulation in poultry $(3,4,5)$. HPAI occurrence in a country brings serious economic consequences and invokes temporary suspension of poultry trade (8). Therefore, confirmation of the disease is subject to notification and immediately triggers countermeasures to prevent its further spread (7).

In 1996, the Guangdong lineage of H5 HPAIV (H5 Gs/Gd) emerged in China and subsequently evolved into multiple genetic clades and genotypes as a result of genetic drift and reassortment $(15,23)$. Since 2008, a specific clade 2.3.4 of H5 Gs/Gd HPAI viruses has undergone frequent reassortments with LPAIV of wild-bird origin and the resulting novel viruses, collectively known as "H5Nx" (H5N2, H5N5, H5N6 and $\mathrm{H} 5 \mathrm{~N} 8$ as differentiated by neuraminidase subtype), show increased adaptation to wild aquatic birds and have spread to other continents, including Europe, Africa and North America (15). In recent years, Europe has experienced repeated outbreaks of HPAIV H5Nx Gs/Gd clade 2.3.4.4 lineage as a result of new introduction of the virus from southeast Asia or Africa, re-emergence of the reassorted virus from the previous epidemic, or the continued endemic circulation of HPAIV H5 (1, 19, 25, 27). In Poland, epidemics of HPAI in poultry have occurred three times: in 2007 (caused by HPAIV H5N1 clade 2.2), in 2016-2017 (caused by HPAIV H5N8 and H5N5 clade 2.3.4.4) and in 2019-2020 (caused by HPAIV H5N8 clade 2.3.4.4) $(24,25,26)$.

In the face of the changing HPAI epidemiological characteristics and the increasingly substantial role of wild birds in the spread of HPAIV into a new area (5), there is a need for continuous improvement of 
emergency preparedness. According to European Union (EU) legislation, Member States must identify risk areas on their territory where there are multiple facilitators of introduction of HPAIV into poultry holdings (6). Relevant risk factors for the introduction of HPAIV into poultry flocks include proximity to wetlands such as swamps and to bodies of water such as ponds, lakes, rivers or the sea where migratory birds, in particular waterfowl and shorebirds, may gather at stop-over sites; poultry holdings being in locations through which migratory birds travel or at which they rest during their movements along the north-eastern and eastern migratory routes into the EU; and keeping poultry in free-range systems where contact between wild birds and poultry cannot be prevented. In turn, the risk factors for the spread of HPAIV between holdings include holdings being in locations with a high density of poultry farming, particularly operations with outdoor access (ducks, geese or free-range layers); frequent movements of vehicles transporting poultry and of persons within and from holdings; and other direct and indirect contacts between holdings being common (6).

The aim of the study was to evaluate the impact of the assumed risk factors, for which quantitative data are available, on HPAI occurrence in commercial Polish flocks during the epidemics which have occurred in recent years. The findings may lead to the improvement of control strategies by fine-tuning risk-based surveillance and advocating for reinforced biosecurity on farms at higher risk of infection.

\section{Material and Methods}

Input data. A set of six risk factors comprising the density of each different farmed species of poultry (chickens, turkeys, ducks and geese) and proximity to areas inhabited by wild birds (water bodies and terrain with high site concentration of wild birds) was considered in the study. Data concerning the density of poultry in each Polish commune $(n=2,477)$ were obtained from the National Statistical Office and the General Veterinary Inspectorate. The locations of bodies of water and sites of wild bird concentrations were obtained from the General Directorate for Environmental Protection.

Disease-related data, i.e. the geographical coordinates of commercial farms on which HPAIV was confirmed during the epidemic in $2007(\mathrm{n}=5)$, the epidemic in 2016-17 $(n=38)$ and the epidemic in 2019-20 $(n=28)$ were derived from the records of the National Reference Laboratory for Avian Influenza in Poland. The locations of 331 randomly selected farms on which HPAIV was not reported during the epidemics were obtained from the General Veterinary Inspectorate. The locations of the farms are presented in Fig. 1.

The spatial distribution of each risk factor in Poland was expressed as a separate raster layer with a raster cell of $250 \times 250 \mathrm{~m}$, using the appropriate package (raster: Geographic Data Analysis and Modeling, R package version 3.4-5) in $\mathrm{R}$ software (version 3.6.1) (21). The input data used to develop the layers include the densities of the four poultry species in each commune, the distance from water bodies and the distance from sites of high wild bird concentration all over the country. Next, the values of each risk factor at the locations of both affected and unaffected farms were extracted. The analysis was developed on the basis of these values. The distributions of the values and the raster layers depicting the spatial distributions of the risk factors in Poland are presented in Table 1.

Analysis. A dataset consisting of 402 observations was considered in the analysis. Each observation includes a binary target variable (HPAI presence/absence) and six quantitative predictor variables (values of each risk factor)

Firstly, the entire dataset was split into a training set ( $70 \%$ of the observations) and a testing set $(30 \%$ of the observations). To predict the probability of HPAI occurrence based on values of predictors (risk factors), three approaches were applied to the training dataset.

Firstly, the logistic regression model of the form $P\left(Y=1 \mid x_{1}, x_{2}, \ldots, x_{6}\right)=\frac{e^{a_{0}+\sum_{i=1}^{6} a_{i} x_{i}}}{1+e^{a_{0}+\sum_{i=1}^{6} a_{i} x_{i}}}$

was used, where $P\left(Y=1 \mid x_{1}, x_{2}, \ldots, x_{6}\right)$ is a conditional probability of the target value of $\mathrm{Y}$ being 1 (HPAI occurrence), given the values of predictors $x_{1}, x_{2}, \ldots, x_{6}$ (risk factors). The regression coefficients $a_{0}, a_{1}, \ldots, a_{6}$ were estimated using maximum likelihood estimation. Logarithm transformation was used for variables with skewed distributions. To construct the final model, the relevant predictors were selected based on a stepwise variable selection method with Akaike Criterion (AIC). The model was developed using the $\mathrm{glm}$ function from the stats package. The levels of multicollinearity among the predictors were assessed using the variance inflation factor (VIF) (13). The linear relationship between the $\log$ odds of the target variable and the predictors was verified through plotting of standardised Pearson residuals against individual predictors (30).

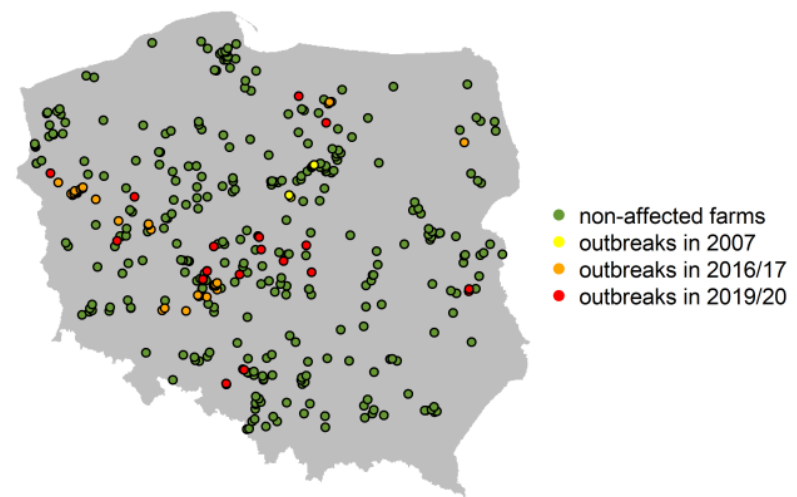

Fig. 1. Locations of affected and unaffected commercial farms. The map was produced using the maps package (maps: Draw Geographical Maps, R package version 3.3.0 (21)) 
Table 1. The list of preliminary selected risk factors with their spatial distribution in Poland and the distribution of values in experimental (from affected farms) and control (from unaffected farms) groups. The maps and graphs were produced using raster and ggplot2 packages (ggplot2: Elegant Graphics for Data Analysis, R package (21)), respectively

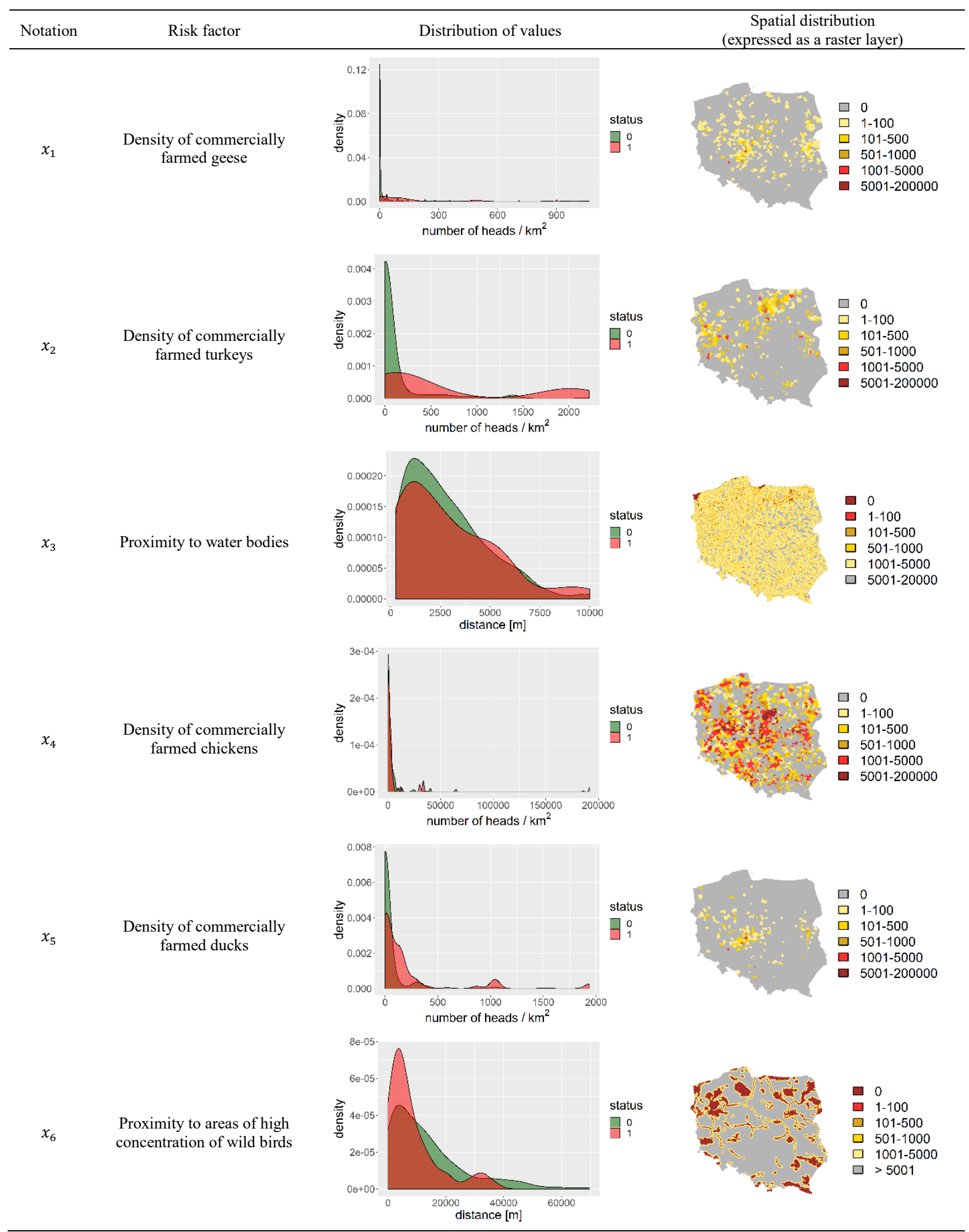

Secondly, the classification tree model was applied to the dataset of the form $(x, Y)=\left(x_{1}, x_{2}, \ldots, x_{6}, Y\right)$, where $Y$ is the target variable (HPAI presence/absence) and $x$ is a vector of the risk factors $x_{1}, x_{2}, \ldots, x_{6}$. The membership of observations in the classes of the binary target variables was predicted based on the values of their predictor variables through fitting the classification tree to the data using the rpart function from the rpart package (rpart: Recursive Partitioning and Regression Trees, R package version 4.1-15) (21). To find accurate 
splits of the set of observations into certain subsets, the Gini index was used (13). In order to determine the optimal level of tree complexity (complexity parameter), 10 -fold cross-validation was performed in three repetitions.

Finally, the random forest model was used. Different decision trees were formed via multiple resampling of the subsets of data (bootstrap sample) and different subsets of predictors from the training dataset. The final probabilities were predicted based on an average of all individual trees. The number of predictors was determined using 10-fold crossvalidation in three repetitions. The number of trees was set to 500. A detailed description of the methods has been published previously (13). The model was developed using the randomForest function from the package of the same name (Classification and Regression by randomForest).

The importance of predictors was estimated using the mean decrease of Gini index in the decision tree and the random forest model using the caret package (caret: Classification and Regression Training, $\mathrm{R}$ package version 6.0-86) (21). The impact of changes in the values of the predictors on the value of probability was determined in each model.

Based on the six raster layers representing the risk factors, the probability of HPAI occurrence was calculated in each raster cell with a size of $250 \times 250 \mathrm{~m}$. For each method, the risk map depicting the spatial distribution of the probability within the country was created using the raster package.

Predictive accuracy of the models was evaluated using the testing dataset. The actual status of each farm (affected or unaffected) was compared with their predicted status, using different cut-off values of probability. The performance of each model was described using the value of accuracy being the number of correctly classified observations divided by the total number of observations. Based on the misclassification matrix, the optimal cut-off value of probability was determined in each model.

\section{Results}

The results of the logistic regression model indicate that the relevant variables (risk factors) include logtransformation of density of turkeys, geese, ducks and chickens. The densities of these species, except chickens, are positively associated with the probability of HPAI occurrence. The detailed statistics for these variables are presented in Table 2.

Table 2. Results of logistic regression model

\begin{tabular}{ccccc}
\hline Notation & Description of the variable & Estimation & $95 \%$ Confidence interval & P value \\
\hline $\log \left(x_{1}+50\right)$ & $\begin{array}{c}\text { log-transformed density of } \\
\text { turkeys }\end{array}$ & 0.973 & {$[0.634,1.355]$} & $9.94 \mathrm{e}^{-08}$ \\
$\log \left(x_{2}+5\right)$ & $\begin{array}{c}\text { log-transformed density of } \\
\text { geese }\end{array}$ & 0.409 & {$[0.113,0.709]$} & $6.92 \mathrm{e}^{-03}$ \\
$\log \left(x_{3}+10\right)$ & $\begin{array}{c}\text { log-transformed density of } \\
\text { ducks }\end{array}$ & 0.447 & {$[0.108,0.791]$} & $9.8 \mathrm{e}^{-03}$ \\
$\log \left(x_{4}+10\right)$ & $\begin{array}{c}\text { log-transformed density of } \\
\text { chickens }\end{array}$ & -0.193 & {$[-0.375,-0.018]$} & $3.31 \mathrm{e}^{-02}$ \\
\hline
\end{tabular}

The form of the final regression model is:

$P(Y)=\frac{e^{-7.88} *\left(x_{1}+50\right)^{0.973} *\left(x_{2}+5\right)^{0.409} *\left(x_{3}+10\right)^{0.447} *\left(x_{4}+10\right)^{-0.193}}{1+e^{-7.88} *\left(x_{1}+50\right)^{0.973} *\left(x_{2}+5\right)^{0.409} *\left(x_{3}+10\right)^{0.447} *\left(x_{4}+10\right)^{-0.193}}$

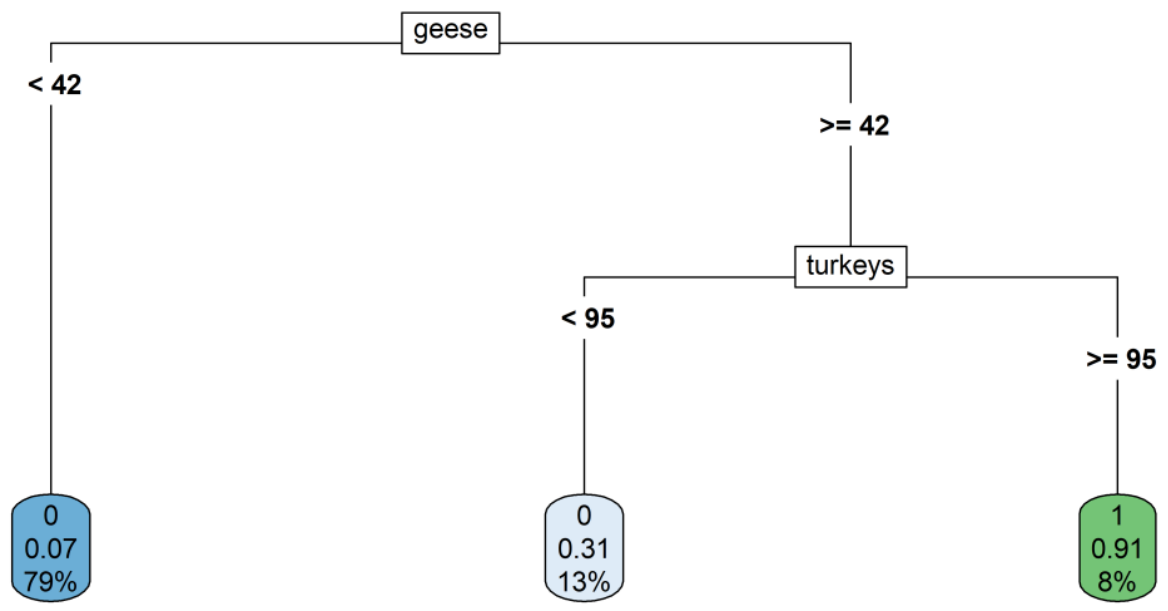

Fig. 2. Results of decision tree model 
Logarithm transformation was used for all predictors. The maximum VIF was lower than 1.87, which indicates the non-existence of high correlations between them. The assumptions of a linear relationship between the log odds of the target variable and the predictors were not fully met, because their distributions were concentrated in one point.

Based on the results of the classification tree model, the areas with both goose density greater than or equal to 42 and turkey density greater than or equal to 95 are at the highest risk. An 8\% share of the observations belonged to these areas, among which HPAIV was confirmed in $91 \%$. The areas with goose density lower than 42 are at low risk. A $79 \%$ of the observations belonged to low-risk areas, among which HPAIV was confirmed in $7 \%$. The detailed predictions of the values of the target variable using the risk factors are presented in Fig. 2.

In the decision tree model, the importance values of the risk factors were 25.54, 21.93, 19.08, 12.19, 3.73 and 0 for densities of turkeys, geese, and ducks, proximity to areas with high concentrations of wild birds, proximity to water bodies and chicken density, respectively, which indicates that the predictions of the model were mainly based on the turkey density, and to a lesser extent, goose and duck densities, proximity to areas with high concentrations of wild birds and proximity to water bodies. The predicted values of probabilities were not affected by the density of chickens. In the random forest model, the importance values of the risk factors were $18.53,17.79,15.01,10.4,9.79$ and 5.93 for densities of turkeys and geese, proximity to areas with high concentrations of wild birds, duck density, proximity to water bodies and chicken density, respectively, which indicates that the predictions of the model were mainly based on the turkey density, and to a lesser extent, goose density, proximity to areas with high concentrations of wild birds, duck density and proximity to water bodies. The prediction of the model was based on chicken density to the least extent. The scaled importance values of the risk factors designated by the decision tree and random forest models are presented in Fig. 3.

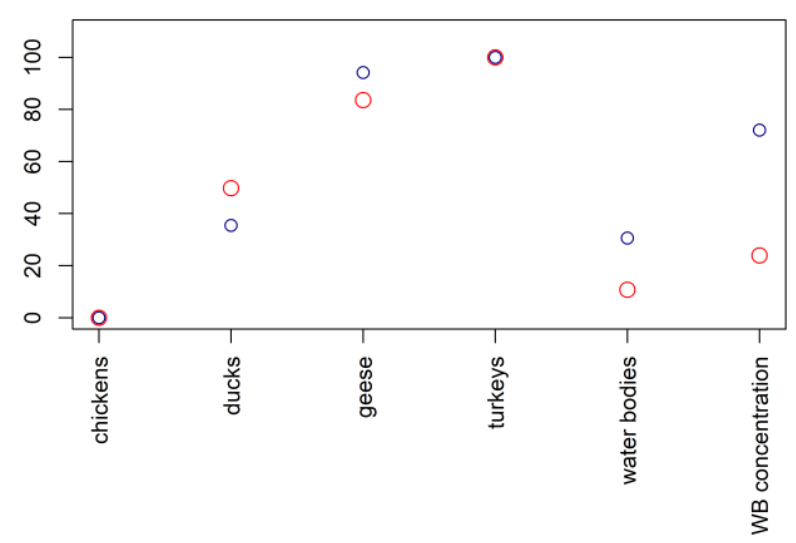

Fig. 3. The importance of considered risk factors designated by the decision tree (red dots) and random forest (blue dots) models. WB wild birds. The importance values were scaled to 0 and 100 (values of 100 and 0 indicated the most important and the least important risk factor in the model, respectively)

The impact of changes in the values of the selected variable on the output value of probability for fixed median values of the other variables is presented in Fig. 4.
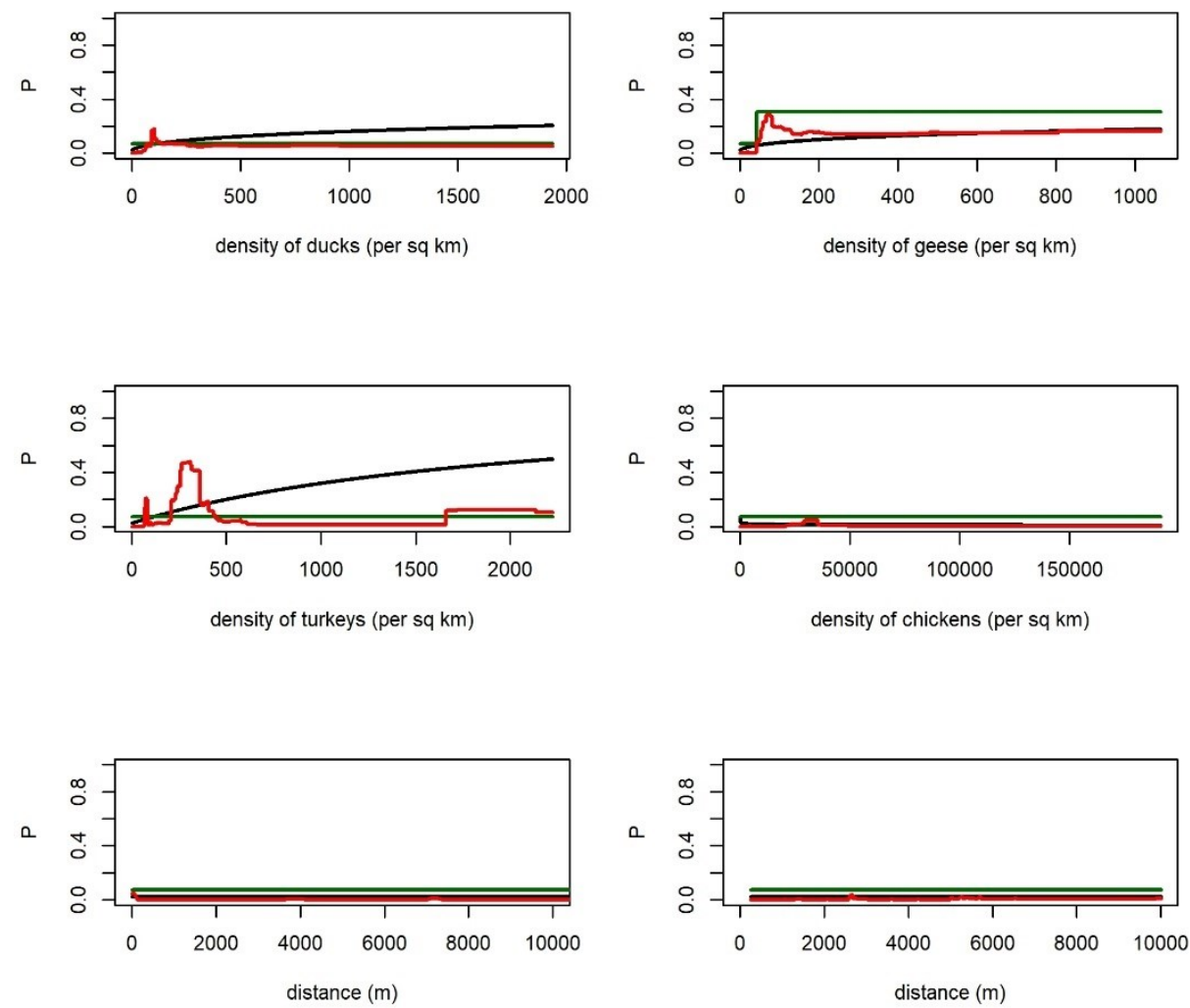

Fig. 4. Impact of changes in the selected variables on the predicted value of probability of HPAI occurrence using different models: logistic regression (black line), decision tree (green line), and random forest (red line). WB - wild birds 
The first, second and third models attained predictive accuracy of 0.8667 (95\% CI $(0.7925$, $0.9218)), 0.875(95 \%$ CI $(0.8022,0.9283))$ and 0.8917 $(95 \%$ CI $(0.8219,0.941))$, respectively. Sensitivity of $80 \%$ was achieved for the cut-off values of 0.05 and 0.12 for the logistic regression and random forest models, respectively. Their corresponding specificities were $52 \%$ and $78 \%$, respectively. Sensitivity of $80 \%$ was not achieved for the decision tree model.

The probability of HPAI occurrence is not equally distributed across the country. Several areas of central, western, eastern and northern Poland are at the highest risk. The spatial distribution of the values of probabilities is presented in Fig. 5.

a)
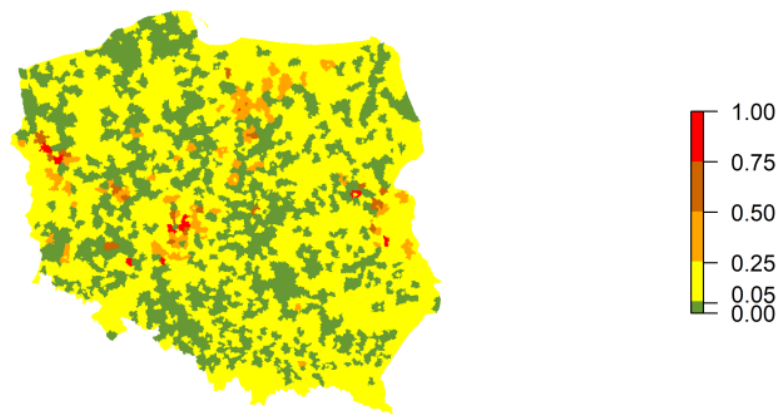

b)

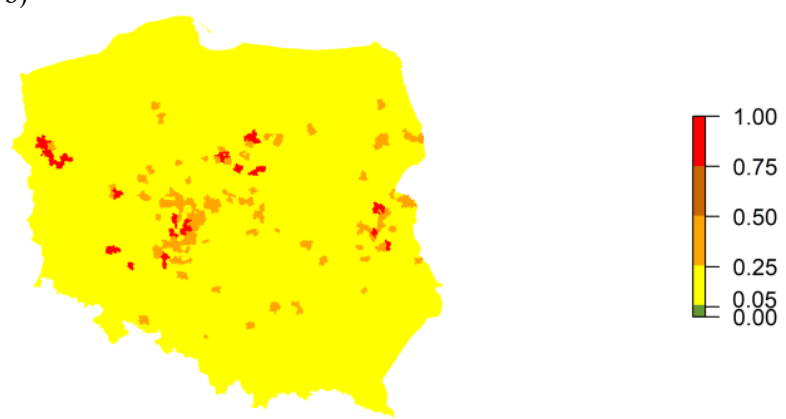

c)

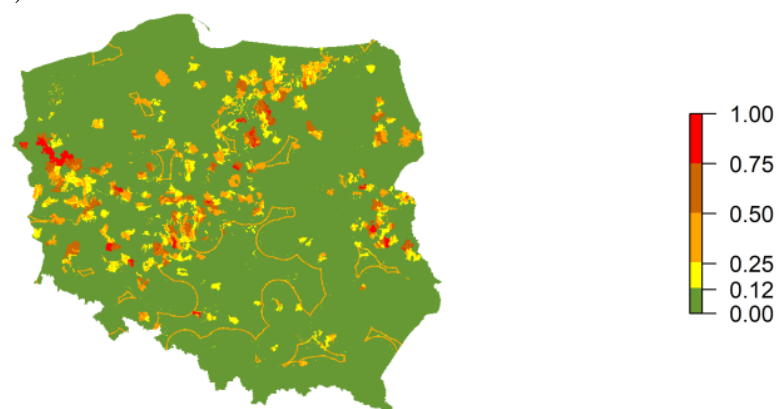

Fig. 5. Spatial distribution of the predicted values of the probabilities using different models: a) logistic regression, b) decision tree, and c) random forest

\section{Discussion}

The results of each model indicated that the densities of turkeys and geese are the most important risk factors. In each model, a slightly different association between the exact number of these poultry species in an area and the values of the probability of
HPAI occurrence was observed (Fig. 4). The other relevant parameter was the density of ducks. Proximity to areas with a high concentration of wild birds was found to be a more important risk factor than proximity to water bodies. Nevertheless, no statistically significant importance of these two parameters was confirmed in the logistic regression model. The presence of chickens in an area hardly had any impact on the value of probability in either the classification tree or random forest models. The logistic regression model indicated, in turn, a negative association between chicken density and the value of the probability (Fig. 4).

The results of our study confirm the impact of the acknowledged risk factors on the occurrence of HPAI outbreaks to a large extent. Similarly to other studies' findings (11), the presence of waterfowl increases the risk of HPAI occurrence due to outdoor access and facilitated contact with wild birds. The proximities to sites with high concentrations of wild birds and to water bodies where migratory birds tend to concentrate were previously demonstrated to be key risk factors for the introduction and further spread of HPAIV H5N1 (28). However, our results did not confirm such a strong impact of these factors on the HPAI occurrence during the recently reported epidemics. It may be related to the more stringent biosecurity and better prevention of contact between wild birds and poultry on commercial farms than on the varying farm types in different countries. On the other hand, turkey density was found to be the most important risk factor. This may be related to the high susceptibility of this species to HPAIV infection. It was shown experimentally that turkeys are $>100$-fold more susceptible to infection with HPAIV $\mathrm{H} 5 \mathrm{~N} 1$ and $\mathrm{H} 7 \mathrm{~N} 1$ subtypes than chickens (2). Additionally, it was also demonstrated that HPAIV H5N8 clade 2.3.4.4 can be successfully transmitted from ducks to turkeys followed by efficient onward transmission among turkeys $(20,22)$.

Interestingly, the presence of commercially farmed chickens in an area did not increase the probability of HPAI outbreak occurrence. The logistic regression model suggested its negative and the other two models its negligible impact. This effect was previously observed also by other authors who studied risk factors for HPAI occurrence in France, China and Indonesia (9, $12,29)$. This phenomenon can be explained by three reasons. First, chickens seem to have lower susceptibility to HPAIV infection than turkeys or ducks, i.e. a much higher virus dose is required to cause infection $(2,14)$. Second, poor or no transmissibility was observed in chickens infected experimentally with HPAIV H5N8 clade 2.3.4.4 despite the high mortality caused by the virus in this species $(14,20)$. Finally, good management and biosecurity practices at commercial chicken farms (including the lack of open-air access) may contribute to the reduced risk of virus introduction onto the holding.

Two types of model - generalized linear and nonlinear - were used to assess the impact of selected risk 
factors on the probability of HPAI occurrence. The logistic regression approach was frequently used to indicate relevant risk factors in other countries $(16,17)$; however, the analysis of the input data suggests the validity of the application of non-linear approaches. Comparing the accuracies of the models, the random forest outperforms the logistic regression in terms of predictive power, in which case $80 \%$ sensitivity with $78 \%$ specificity was attained. It may be explained by non-linear associations or the presence of interactions between risk factors. Comparing the two non-linear models in turn, the random forest was much more accurate than the decision tree. This is observed very often, because the former method combines the output of multiple (randomly created) decision trees to generate the final output. As the decision tree model's results are simpler to interpret than those of the random forest model, they were presented only for visualisation of the impact of particular risk factors, but not as an alternative or competitive method. In view of this, the random forest approach should be the method of choice to predict the areas at increased risk of HPAI occurrence in Poland.

None of the models allowed very high $(>80 \%)$ sensitivity to be obtained with acceptable specificity. A $20 \%$ share of observations from the testing dataset was incorrectly classified - the predicted status of farms (affected or unaffected) did not reflect their actual status, even using a low cut-off value. This may suggest the impact of other risk factors than those included in our study, and this aspect should be further investigated. Nevertheless, the required data are often missing or their expression in quantitative terms is not always feasible. For instance, as indicated in other studies, the association of HPAI occurrence with anthropogenic risk factors is undeniable because virus spread by personnel and fomites is known to be possible $(16,18)$. The impact of these risk factors was previously estimated by population density in the county around the farm or in the area adjacent to the farm. However, our study focuses only on commercial poultry farming where any visits to the premises should be restricted to a minimum. Therefore, the more relevant risk factor is related to farming practices and biosecurity level on a farm (17). Nevertheless, quantifying the effect of these was also not feasible due to the lack of accurate and fully reliable data.

This is the second spatial analysis developed to predict the areas at risk of HPAI occurrence in commercial flocks in Poland. The first analysis combined knowledge mined from the literature and opinions of Polish experts in the field of epidemiology and poultry diseases (10). Due to the development of the first model preceding the largest HPAI epidemic in Poland, data related to the locations of HPAI outbreaks were unavailable. After more extensive epidemic data became available, the obtained data were only used for the model verifications. Therefore, the first study reflected the arbitrary evaluations of each expert related to the key risk factors and their impact on HPAI occurrence in Poland.

Comparing the results of the study reported herein with those of the pre-epidemic one, it can be noted that the overlapping areas at high risk mainly include parts of the Lubuskie, Lubelskie, Łódzkie, and Wielkopolskie provinces. As indicated by the experts, the common risk factors included density of turkeys, domestic waterfowl and proximity to areas with high concentrations of wild birds. Each of these risk factors was assumed to be positively associated with the areas at risk of HPAI occurrence.

The number of observations (reported outbreaks of HPAI) included in the model was relatively small. Inclusion of new cases would improve the quality of the model. Therefore, if the epidemic reoccurs in the country, the experimental group should be extended. The data related to the densities of different poultry species considered in the analysis should be updated regularly.

The models developed here can be a valuable source of information for different groups of stakeholders, including poultry owners and risk managers. The results can also lead to the improvement of targeted surveillance in Poland.

Conflict of Interests Statement: The authors declare that there is no conflict of interests regarding the publication of this article.

Financial Disclosure Statement: This study was funded by the "KNOW" (Leading National Research Centre) Scientific Consortium "Healthy Animal - Safe Food", Ministry of Science and Higher Education resolution no. 05-1/KNOW2/2015.

Animal Rights Statement: Not applicable.

\section{References}

1. Alarcon P., Brouwer A., Venkatesh D., Duncan D., Dovas C.I., Georgiades G., Monne I., Fusaro A., Dan A., Śmietanka K., Ragias V., Breed A.C., Chassalevris T., Goujgoulova G., Hjulsager C.K., Ryan E., Sánchez A., Niqueux E., Tammiranta N., Zohari S., Stroud D.A., Savić V., Lewis N.S., Brown I.H.: Comparison of 2016-17 and Previous Epizootics of Highly Pathogenic Avian Influenza H5 Guangdong Lineage in Europe. Emerg Infect Dis 2018, 24, 2270-2283, doi:10.3201/ eid2412.171860.

2. Aldous E.W., Seekings J.M., McNally A., Nili H., Fuller C.M., Irvine R.M., Alexander D.J., Brown I.H.: Infection dynamics of highly pathogenic avian influenza and virulent avian paramyxovirus type 1 viruses in chickens, turkeys and ducks. Avian Pathol 2010, 39, 265-273, doi: 10.1080/03079457. 2010.492825 .

3. Alexander D.J.: An overview of the epidemiology of avian influenza. Vaccine 2007, 25, 5637-5644, doi: 10.1016/j.vaccine. 2006.10.051.

4. Alexander D.J., Capua I.: Avian influenza in poultry. World Poultry Sci J 2008, 64, 513-532, doi: 10.1017/ S0043933908000184. 
5. Bodewes R., Kuiken T.: Changing Role of Wild Birds in the Epidemiology of Avian Influenza A Viruses. Adv Virus Res 2018, 100, 279-307, doi: 10.1016/bs.aivir.2017.10.007.

6. Commission of the European Communities: Commission Implementing Decision (EU) 2018/1136 of 10 August 2018 on risk mitigation and reinforced biosecurity measures and early detection systems in relation to the risks posed by wild birds for the transmission of highly pathogenic avian influenza viruses to poultry. OJ L 2018, 205, 61, 14.8.2018, pp. 48-53.

7. Council of the European Union: Council Directive 2005/94/EC of 20 December 2005 on Community measures for the control of avian influenza and repealing Directive 92/40/EEC. OJ L 2006, 10, 49, 14.1.2006, pp. 16-65.

8. Domenech J., Lubroth J., Eddi C., Martin V., Roger F.: Regional and international approaches on prevention and control of animal transboundary and emerging diseases. Ann N Y Acad Sci 2006, 1081, 90-107, doi: 10.1196/annals.1373.010.

9. Fang L-Q., de Vlas S.J., Liang S., Looman C.W., Gong P., Xu B., Yan L., Yang H., Richardus J.H., Cao W-C.: Environmental factors contributing to the spread of $\mathrm{H} 5 \mathrm{~N} 1$ avian influenza in mainland China. PLoS One 2008, 3, e2268, doi: 10.1371/ journal.pone.0002268.

10. Gierak A., Bocian Ł., Śmietanka K.: Identification of Areas at Increased Risk of Highly Pathogenic Avian Influenza Occurrence in Commercial Poultry in Poland. Avian Dis 2019, 63, 257-262, doi: 10.1637/12005-112718.1.

11. Gilbert M., Pfeiffer D.U.: Risk factor modelling of the spatiotemporal patterns of highly pathogenic avian influenza (HPAIV) H5N1: a review. Spat Spatiotemporal Epidemiol 2012, 3, 173-183, doi: 10.1016/j.sste.2012.01.002.

12. Guinat C., Artois J., Bronner A., Guérin J-L., Gilbert M., Paul M.C.: Duck production systems and highly pathogenic avian influenza H5N8 in France, 2016-2017. Sci Rep 2019, 9, 61-77, doi: 10.1038/s41598-019-42607-x.

13. James G., Witten D., Hastie T., Tibshirani R.: An Introduction to Statistical Learning with Applications in R. Springer Science+Business Media, New York, 2013.

14. Leyson C., Youk S-S., Smith D., Dimitrov K., Lee D-H., Larsen L.E., Swayne D.E., Pantin-Jackwood M.J.: Pathogenicity and genomic changes of a 2016 European H5N8 highly pathogenic avian influenza virus (clade 2.3.4.4) in experimentally infected mallards and chickens. Virology 2019, 537, 172-185, doi: 10.1016/j.virol.2019.08.020.

15. Lycett S.J., Duchatel F., Digard P.: A brief history of bird flu. Phil Trans R Soc B 2019, 374, 20180257, doi: 10.1098/rstb.2018.0257.

16. Métras R., Stevens K.B., Abdu P., Okike I., Randolph T., Grace D., Pfeiffer D.U., Costard S.: Identification of potential risk factors associated with highly pathogenic avian influenza subtype H5N1 outbreak occurrence in Lagos and Kano States, Nigeria, during the 2006-2007 epidemics. Transbound Emerg Dis 2013, 60, 87-96, doi: 10.1111/j.1865-1682.2012.01322.x.

17. Osmani M.G., Thornton R.N., Dhand N.K., Hoque M.A., Milon S.M., Kalam M.A., Hossain M., Yamage M.: Risk factors for highly pathogenic avian influenza in commercial layer chicken farms in Bangladesh during 2011. Transbound Emerg Dis 2014, 61, e44-51, doi: 10.1111/tbed.12071.

18. Paul M., Tavornpanich S., Abrial D., Gasqui P., Charras-Garrido M., Thanapongtharm W., Xiao X., Gilbert M., Roger F., Ducrot C.: Anthropogenic factors and the risk of highly pathogenic avian influenza H5N1: prospects from a spatial-based model. Vet Res 2010, 41, 28, doi: 10.1051/vetres/2009076.

19. Poen M.J., Venkatesh D., Bestebroer T.M., Vuong O., Scheuer R.D., Munnink B.B.O., de Meulder D., Richard M., Kuiken T., Koopmans M.P.G., Kelder L., Kim Y-J., Lee Y-J.,
Steensels M., Lambrecht B., Dan A., Pohlmann A., Beer M., Savić V., Brown I.H., Fouchier R.A.M., Lewis N.S.: Co-circulation of genetically distinct highly pathogenic avian influenza A clade 2.3.4.4 (H5N6) viruses in wild waterfowl and poultry in Europe and East Asia, 2017-18. Virus Evol 2019, 5, vez004, doi: 10.1093/ve/vez004.

20. Puranik A., Slomka M.J., Warren C.J., Thomas S.S., Mahmood S., Byrne A.M.P., Ramsay A.M., Skinner P., Watson S., Everett H.E., Núñez A., Brown I.H., Brookes S.M.: Transmission dynamics between infected waterfowl and terrestrial poultry: Differences between the transmission and tropism of H5N8 highly pathogenic avian influenza virus (clade 2.3.4.4a) among ducks, chickens and turkeys. Virology 2020, 541, 113-123, doi: 10.1016/j.virol. 2019.10.014

21. R Core Team: R: A language and environment for statistical computing. R Foundation for Statistical Computing, Vienna, 2016, 2019, 2020 .

22. Slomka M.J., Puranik A., Mahmood S., Thomas S.S., Seekings A.H., Byrne A.M.P., Núñez A., Bianco C., Mollett B.C., Watson S., Brown I.H., Brookes S.M.: Ducks Are Susceptible to Infection with a Range of Doses of H5N8 Highly Pathogenic Avian Influenza Virus (2016, Clade 2.3.4.4b) and Are Largely Resistant to Virus-Specific Mortality, but Efficiently Transmit Infection to Contact Turkeys. Avian Dis 2019, 63, 172-180, doi: 10.1637/11905-052518-Reg.1.

23. Smith G.J., Donis R.O., World Health Organization/World Organisation for Animal Health/Food and Agriculture Organization (WHO/OIE/FAO) H5 Evolution Working Group.: Nomenclature updates resulting from the evolution of avian influenza $\mathrm{A}(\mathrm{H} 5)$ virus clades 2.1.3.2a, 2.2.1, and 2.3.4 during 2013-2014. Influenza Other Respir Viruses 2015, 9, 271-276, doi: 10.1111/irv.12324.

24. Śmietanka K., Fusaro A., Domańska-Blicharz K., Salviato A., Monne I., Dundon W.G., Cattoli G., Minta Z.: Full-Length Genome Sequencing of the Polish HPAI H5N1 Viruses Suggests Separate Introductions in 2006 and 2007. Avian Dis 2010, 54, 335-339, doi: 10.1637/8782-040109-ResNote.1.

25. Święton E., Fusaro A., Shittu I., Niemczuk K., Zecchin B., Joannis T., Bonfante F., Śmietanka K., Terregino C.: Sub-Saharan Africa and Eurasia Ancestry of Reassortant Highly Pathogenic Avian Influenza A(H5N8) Virus, Europe, December 2019. Emerg Infect Dis 2020, 26, 1557-1561, doi:10.3201/eid2607.200165.

26. Święton E., Śmietanka K.: Phylogenetic and molecular analysis of highly pathogenic avian influenza $\mathrm{H} 5 \mathrm{~N} 8$ and $\mathrm{H} 5 \mathrm{~N} 5$ viruses detected in Poland in 2016-2017. Transbound Emerg Dis 2018, 65, 1664-1670, doi: 10.1111/tbed.12924.

27. Venkatesh D., Brouwer A., Goujgoulova G., Ellis R., Seekings J., Brown I.H., Lewis N.S.: Regional Transmission and Reassortment of 2.3.4.4b Highly Pathogenic Avian Influenza (HPAI) Viruses in Bulgarian Poultry 2017/18. Viruses 2020, 12, 605, doi: 10.3390/v12060605.

28. Ward M.P., Maftei D.N., Apostu C.L., Suru A.R.: Association between outbreaks of highly pathogenic avian influenza subtype H5N1 and migratory waterfowl (family Anatidae) populations. Zoonoses Public Health 2009, 56, 1-9, doi: 10.1111/j.18632378.2008.01150.x.

29. Yupiana Y., de Vlas S.J., Adnan N.M., Richardus J.H.: Risk factors of poultry outbreaks and human cases of H5N1 avian influenza virus infection in West Java Province, Indonesia. Int J Infect Dis 2010, 14, e800-805, doi: 10.1016/j.ijid.2010.03.014.

30. Zhang Z.: Residuals and regression diagnostics: focusing on logistic regression. Ann Transl Med 2016, 4, 195, doi: 10.21037/atm.2016.03.36. 